\title{
In vivo antimalarial potential of tinospora crispa miers in mice and identification of the bioactive compound
}

\begin{abstract}
Background: Malaria is a serious disease that is causing huge toll on human health. The parasites causing this disease have developed resistance toward the current mainstream drugs. Tinospora crispa Miers is a well-known ethnopharmacological plant of Malaysia and has been traditionally used to treat malaria, fever, and other parasite-related illnesses. Objective: The present study investigated the potential of the crude methanol extract and antiparasitic fractions of $\mathrm{T}$. crispa stem to exert antimalarial activity against Plasmodium berghei. Materials and Methods: The potent antiparasitic fractions of $\mathrm{T}$. crispa, F4, and F5 were isolated in the previous study against Toxoplasma gondii. The same antiparasitic fractions along with crude methanol extract of different doses $(10,50,100 \mathrm{mg} / \mathrm{kg} \mathrm{b}$. w.) were employed in the present study to determine the antimalarial activity against $P$. berghei ANKA strain. The survival curves of the treated mice were plotted by employing the log-rank (Mantel-Cox) test. The chemical composition of the most potent fraction F5 was determined spectrometrically using electrospray ionization-mass spectrometry (MS). Results: In a murine P. berghei model, fraction F5 displayed the highest parasitemia suppression effects compared to the crude methanol extract and other fraction. Subsequent chemical analysis by MS on fraction F5 has led to the tentative identification of 13-hydroperoxyoctadeca-9, 11dienoic acid (13[S]-HPODE) compound. Conclusion: The crude methanol extract of T. crispa and its fraction F5 possess potent antimalarial activities, and the tentative discovery of the 13(S)HPODE bioactive compound may serve as a precursor for developing a semisynthetic antiparasitic drug with enhanced efficacy and low toxicity.
\end{abstract}

Keyword: 13-hydroperoxyoctadeca-9; 11-dienoic acid; Antimalarial; Parasitemia; Plasmodium berghei, Tinospora crispa Miers 